\title{
Estado da Arte dos Doentes \\ com Cancro do Pulmão \\ de Não Pequenas Células: Doença \\ Avançada e Mutação EGFR Positiva
}

\section{Advanced EGFR Mutation-Positive Non-Small-Cell Lung Cancer: State of the Art}

Bárbara Parente ${ }^{1}$

\section{RESUMO}

O aparecimento de terapêuticas dirigidas, trouxe na última década uma enorme esperança para os doentes com cancro do pulmão. Os inibidores dos recetores do fator de crescimento epidérmico (EGFR), erlotinib e gefitinib (inibidores de tirosina quinase TKI), estiveram na vanguarda das mudanças da prática de tratamento para doença avançada do cancro do pulmão de não pequenas células (CPNPC), nos últimos 10 anos, com a descoberta de mutações EGFR que conferem sensibilidade a inibidores da tirosina quinase no CPNPC.

O ano de 2004, marcou o início da era da medicina de precisão para o cancro do pulmão. Os estudos randomizados de fase III investigaram o papel de dois inibidores de EGFR-TKI, gefitinib e erlotinib, como tratamento de primeira linha em comparação com a quimioterapia à base de platina, em doentes com CPNPC doença avançada com mutações de ativação do EGFR.

Um TKI de segunda geração (afatinib) encontra-se já aprovado pela EMA com a mesma indicação após resultados dos clínicos do Lux Lung 3 e 6. No entanto, os doentes com mutação EGFR positiva, regra geral, progridem após 10-12 meses de tratamento, necessitando de novas opções terapêuticas. Inibidores de $3^{a}$ geração entretanto desenvolvidos (dirigidos à mutação de resistência do T790M, e outras, após rebiópsia), demostraram uma notável eficácia, em doentes já tratados e com um perfil de toxicidade ainda menor que os TKI de $1^{\text {a e }} 2^{\mathrm{a}}$ geração

PALAVRAS-CHAVE: Carcinoma Pulmonar de Células Não Pequenas; Inibidores dos Recetores do Fator de Crescimento Epidérmico 


\section{ABSTRACT}

The emergence of targeted therapies, brought in the last decade a large hope for patients with lung cancer. EGFR tyrosine kinase inibitors, (TKI) erlotinib and gefitinib, changed the last 10 years the practice of treatment for advanced non-small-cell lung cancer (NSCLC) with the discovery of EGFR mutations that confer sensitivity to tyrosine kinase inhibitors in NSCLC. The year of 2004 marked the Era of Precision Medicine for lung cancer. The studies randomized phase III investigated the role of two inhibitors of EGFR-TKI, gefitinib and erlotinib, as the treatment of first line compared with platinum-based chemotherapy in NSCLC patients with advanced disease, EGFR activating mutations. A second generation TKI (afatinib) is already approved for the same indication (EMA) with the good results of the clinical trials, Lux Lung 3 and 6. However patients with EGFR positive mutation, usually have a progressive disease after 10-12 months, with need of new treatment options. Inhibitors third generation however developed (for resistance mutation, T790M and others, after rebiopsy), demonstrated a remarkable efficacy in patients already treated, and with a toxicity profile better than TKI of $1^{\text {st }}$ and $2^{\text {nd }}$ generation.

KEYWORDS: Carcinoma, Non-Small-Cell Lung; Receptor, Epidermal Growth Factor

Com o aparecimento de terapêuticas dirigidas, assistimos, na última década, a uma esperança acrescida para os doentes com cancro do pulmão, bem como para todos os envolvidos nesta luta, nomeadamente médicos e cientistas, face às baixas taxas de sobrevida aos 5 anos nos doentes com cancro do pulmão.

Os inibidores dos recetores do fator de crescimento epidérmico (EGFR), erlotinib e gefitinib (inibidores de tirosina quinase TKI) estiveram na vanguarda das mudanças do tratamento para doença avançada do cancro do pulmão de não pequenas células (CPNPC) nos últimos 10 anos, devido à descoberta de mutações EGFR que conferem sensibilidade a estes fármacos no CPNPC, particularmente em doentes com adenocarcinoma do pulmão, mulheres e não fumadores ou ex-fumadores. ${ }^{1}$

O ano de 2004 marcou o início da medicina de precisão para o cancro do pulmão, resultante do esforço efetuado por muitos investigadores, para melhor definir subgrupos moleculares deste tumor e identificar novos alvos terapêuticos, definindo mecanismos de sensibilidade e resistência às terapêuticas específicas. Estudos randomizados de fase III investigaram o papel de dois inibidores de EGFR-TKI, o gefitinib² e o erlotinib ${ }^{3,4}$ como tratamento de primeira linha em comparação com a quimioterapia à base de platina, em doentes com CPNPC doença avançada e mutações de ativação do EGFR. Em todos estes ensaios, o objetivo principal foi alcançado, evidenciando uma melhoria significativa na sobrevida livre de progressão (PFS) e na taxa de resposta global (RR). A sobrevida global (OS), quando disponível, foi semeIhante entre os TKI e o braço de quimioterapia, devido à elevada percentagem de cross-over no tratamento. No entanto, a OS atingiu cerca de 30 meses, resultados nunca antes observados em ensaios dirigidos a doentes com CPNPC doença avançada. $\bigcirc$ estudo com erlotinib versus quimioterapia (EURTAC) 4 foi o primeiro com um ITC di- rigido a uma população europeia com CPNPC doença avançada com mutações do EGFR (deleção do exão 19 ou mutação L858R no exão 21). Neste ensaio, o erlotinib, na dose oral de 150 mg por dia, foi comparado com dupletos à base de platina em doentes elegíveis, tendo sido significativamente melhor do que a quimioterapia na PFS (objetivo principal do estudo), com 9,7 contra 5,2 meses, respetivamente (HR 0,37; IC 95\% 0,25-0,54).

Os frutos desta investigação são agora visíveis no dia-a- dia do clínico que trata os doentes com cancro do pulmão: um impacto muito significativo relativamente aos resultados obtidos pelo uso da quimioterapia. Por outro lado, o perfil de tolerância e a necessidade de visitas obrigatórias foram reduzidos para os doentes que detêm as mutações ativadoras de um mecanismo oncogénico que Ihes permite beneficiar dessas terapêuticas.

As mutações mais comuns do gene EGFR utilizadas para orientar decisões de tratamento em CPNPC situam-se nas deleções no exão 19 do EGFR e ponto de mutação específica L858R no exão 21. Vários métodos conseguem identificar outras mutações ativadoras menos comuns, nomeadamente no exão 18 e exão 20, algumas delas de resistência, sendo entre estas a mais frequente a mutação de resistência a T790M no exão 20, conferindo vantagem competitiva aos TKI e impedindo ou retardando a proliferação tumoral.

Utilizando um mecanismo idêntico, a translocação do ALK, quando presente, pode ser bloqueada por um inibidor específico, o crisotinib, resultando na paragem de proliferação tumoral. 5

Faz hoje parte integrante do estudo destes doentes a realização de testes moleculares que permitem determinar se o tumor tem uma mutação que pode conduzir ao uso de novas e melhores terapêuticas dirigidas.

Em Portugal, os estudos efetuados até à data apontam 
para uma taxa de mutação do EGFR de cerca de $13 \%^{6}$ e uma taxa de translocação do ALK de 5-7\%. Na generalidade, estes doentes (EGFR + ou ALK +) são tratados, hoje em dia, nos hospitais portugueses, com os inibidores específicos, daí resultando um benefício acentuado na resposta ao tratamento, PFS, sobrevida global, tolerância e comodidade terapêutica (num grupo de aproximadamente 20\% de doentes). Nos restantes casos, ainda a maioria, a quimioterapia baseada no subtipo histológico continua a ser a melhor opção terapêutica.

De acordo com os resultados anteriormente referidos, o gefitinib foi o primeiro TKI a receber a aprovação para tratamento de primeira linha em doentes com mutação EGFR em 2008. O mesmo aconteceu para o erlotinib em 2013, com base na evidência científica, sendo aprovado como agente único versus quimioterapia para o tratamento de primeira linha em doentes com CPNPC doença avançada em tumores com mutações do EGFR.

Recentemente, verificou-se o aparecimento de TKI de segunda geração como o afatinib e dacomitinib. Tendo em conta os estudos Lux - Lung 3 e 67,8 com o afatinib, também este fármaco encontra-se aprovado pela Agência Europeia de Medicamentos (EMA). Os ensaios mostraram ainda uma melhor resposta para os doentes com mutação no exão 19, confirmados nos estudos asiáticos com gefitinib (IPASS) ${ }^{2}$ e erlotinib (OPTIMAL), ${ }^{3}$ bem como no estudo europeu EURTAC. ${ }^{4}$ No que concerne ao dacomitinib, os estudos encontram-se em curso nomeadamente com gefitinib, (ARCHER 1050 - fASE III) de forma a demonstrar a mais-valia do seu uso em primeira linha no CPNPC doença avançada.

De acordo com os trabalhos publicados até esta data, o Lux - Lung -79 é o primeiro estudo prospetivo que avalia

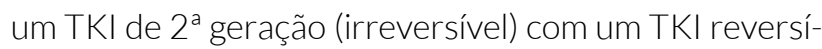
vel, no caso o gefitinib, para doentes com CPNPC doença avançada em primeira linha em doentes mutados em ambos os grupos asiáticos e não asiáticos. $\bigcirc$ afatinib melhorou significativamente a PFS bem como o tempo até ao fim do tratamento. $O$ número de doentes que encontraram taxa de resposta objetiva foi também significativamente maior com afatinib do que com o gefitinib. Os efeitos adversos foram bem tolerados com ambos os fármacos com baixas taxas de descontinuação. A diarreia e o rash estiveram mais associados ao afatinib, enquanto as alterações hepáticas e a pneumonite de hipersensibilidade mais relacionadas com o gefitinib. A qualidade de vida mostrou iguais resultados com os dois fármacos. A melhoria na eficácia foi observada tanto em doentes com mutação no exão 19, como na supressão de mutações no Leu858Arg. As evidências disponíveis no Lux-Lung 7 indicam que o bloqueio ErbB irreversível com afatinib pode ser mais efetivo do que a inibição EGFR reversível no tratamento de CPNPC com mutação EGFR-positiva. Os resultados sugerem, ainda, que a primeira geração e segunda geração de TKI não são permutáveis, implicando que o mecanismo mais amplo e irreversível da ação do afatinib, em comparação com gefitinib, pode ter conduzido a um melhor controlo tumoral.

Sabe-se, no entanto, que na maioria dos doentes com mutação EGFR, após cerca de 10-12 meses de tratamento, ocorre progressão com necessidade de novas opções terapêuticas. Utilizando o conhecimento obtido pela rebiopsia dos tumores que progridem sob tratamento com inibidores de TKI dos tumores e com o uso de técnicas mais sensíveis, tais como sequenciação de última geração, e uso potencial de amostras de sangue, pode-se tornar mais simples a obtenção de material para prosseguimento de estudo. Verificou-se que cerca de 50-55\% dos doentes adquirem mutação de resistência do T790M no exão 20, sendo esta a mais frequente mutação de resistência. Os novos inibidores desta mutação (inibidores de $3^{a}$ geração) entretanto desenvolvidos, demostraram uma notável eficácia (60\% de taxa de reposta e 90\% de controlo da doença com 12 a 14 meses de tempo livre de progressão) em doentes já tratados e com um perfil de toxicidade ainda menor que os TKI de $1^{\mathrm{a}}$ e $2^{\mathrm{a}}$ geração. ${ }^{10}$

Assim, num relance sobre a melhoria de resultados no CPNPC doença avançada nos últimos dez anos, e apesar de o paradigma da sobrevida aos 5 anos não se ter ainda alterado, constata-se que as sobrevidas médias passaram de 4 meses (na década de 80) para 17 meses no subtipo adenocarcinoma não mutado. ${ }^{11}$ No grupo dos doentes com mutação EGFR+, em que o adenocarcinoma é o tumor principal, a sobrevida média pode ir até aos 24 meses, o que se afigura da maior relevância quando se fala de doentes com cancro do pulmão doença avançada.

CONFLITOS DE INTERESSE: Os autores declaram a inexistência de conflitos de interesse na realização do trabalho.

FONTES DE FINANCIAMENTO: Não existiram fontes externas de financiamento para a realização deste artigo.

\section{REFERÊNCIAS}

1. Shepherd FA, Pereira JR, Ciuleanu T, Tan EH, Hirsh V, Thongprasert S. Erlotinib in previously treated non-small-cell lung cancer. N Engl J Med. 2005;353:123-32.

2. Mok TS, Wu YL, Thongprasert S, Chu DT, Saijo N, Sunpaweravong P, et al. Gefitinib or carboplatin-paclitaxel in pulmonary adenocarcinoma. N Engl J Med. 2009;361:947-57.

3. Zhou C, Wu YL, Chen G, Feng J, Liu XQ, Wang C, et al. Erlotinib versus chemotherapy as first-line treatment for patients with advanced EGFR mutation-positive non-small-cell lung cancer (OPTIMAL, CTONG-0802): a multicentre, open-label, randomised, phase 3 study. Lancet Oncol. 2011;12: 735-42. 
4. Rosell R, Carcereny E, Gervais R, Vergnenegre A, Massuti B, Felip E, et al. Erlotinib versus standard chemotherapy as first-line treatment for European patients with advanced EGFR mutation-positive non-small-cell lung cancer (EURTAC): a multicentre, open-label, randomised phase 3 trial. Lancet Oncol. 2012;13:239-46.

5. Solomon BJ, Mok T, Kim DW, Wu YL, Nakagawa K, Mekhail T, et al. First-line crizotinib versus chemotherapy in ALK-positive lung cancer. N Engl J Med. 2014;371:2167-77.

6. Castro AS, Parente B, Gonçalves I, Antunes A, Barroso A, Conde $\mathrm{S}$, et al. Epidermal growth factor receptor mutation study for 5 years, in a population of patients with non-small-cell lung cancer. Rev Port Pneumol. 2013;19:7-12.

7. Sequist LV, Yang JC, Yamamoto N, O'Byrne K, Hirsh V, Mok T, et al. Phase III study of afatinib or cisplatin plus pemetrexed in patients with metastatic lung adenocarcinoma with EGFR mutations. J Clin Oncol. 2013;31:3327-34.

8. Wu YL, Zhou C, Hu CP, Feng J, Lu S, Huang Y,et al. Afatinib versus cisplatin plus gemcitabine for first-line treatment of Asian patients with advanced non-small-cell lung cancer harbouring EGFR mutations (LUX-Lung 6): an open-label, randomised phase 3 trial. Lancet Oncol. 2014;15:213-22.
9. Park K, Tan EH, O’Byrne K, Zhang L, Boyer M, Mok T, et al. Afatinib versus gefitinib as first-line treatment of patients with EGFR mutation-positive non-small-cell lung cancer (LUX-Lung 7): a phase $2 \mathrm{~B}$, open-label, randomised controlled trial. Lancet Oncol. 2016;17:577-89.

10. Janne PA, Yang JC, Kim DW, Planchard D, Ohe Y, Ramalingam SS, et al. AZD9291 in EGFR inhibitor-resistant non-small-cell lung cancer. N Engl J Med. 2015;372:1689-99.

11. Paz-Ares LG, de Marinis F, Dediu M, Thomas M, Pujol JL, Bidoli P, et al. PARAMOUNT: Final overall survival results of the phase III study of maintenance pemetrexed versus placebo immediately after induction treatment with pemetrexed plus cisplatin for advanced non-squamous non-small-cell lung cancer. J Clin Oncol. 2013;31:2895-902. 